\title{
Simulación de Convertidores de Potencia en Sistemas Eólicos
}

Rui Melício y Victor M.F. Mendes

Instituto Superior de Engenharia de Lisboa, Departamento de Engenharia Electrotécnica e Automação, Rua Conselheiro Emídio Navarro, 1949-014 Lisboa-Portugal

(e-mail: ruimelicio@gmail.com, vfmendes@isel.pt)

\section{Resumen}

En el presente trabajo se hace un análisis actual de la capacidad instalada de producción de energía eléctrica utilizando fuentes de energía renovable en Portugal y se analiza la situación actual relacionada con convertidores electrónicos de potencia. Además el trabajo aporta información sobre la simulación de topologías de convertidores de potencia en los sistemas de energía eólica, los convertidores usuales de dos niveles, el convertidor multinivel y el convertidor matricial. Se usa el método de la modulación de los pulsos de disparo mediante vectores espaciales de tensión y el control por modo de deslizamiento. Esta simulación permite concluir que las ondas de tensión y corriente en los convertidores de dos etapas AC/DC/AC se presentan con mejor comportamiento armónico que en el convertidor de una etapa AC/AC.

Palabras clave: energía eólica, convertidor dos niveles, convertidor multinivel, convertidor matricial

\section{Simulation of Power Converters for Wind Energy Systems}

\begin{abstract}
This paper presents an analysis of the present installed electric power capacity of renewable energy power systems in Portugal, and analyzes the present state of power converters applied to wind farms. Also, this paper presents a simulation of a wind energy system with the usual topology for power converters, a two-levels converter, a multi-level converter and a matrix converter, using pulse modulation by space vector modulation. The simulation allows concluding that the waves for the electric voltage and the current in the converters $A C / D C / A C$ have better performance than in the matrix converter AC/AC.
\end{abstract}

Keywords: wind power, two-levels converter, multi-level converter, matrix converter 


\section{INTRODUCCIÓN}

En la gran mayoría de los países, entre los cuales se encuentra Portugal, los combustibles fósiles son utilizados en porcentaje significativo para satisfacer sus necesidades energéticas. La producción de energía eléctrica por medio de centrales térmicas convencionales tiene efectos nocivos sobre el medio ambiente, especialmente originando alteraciones climáticas debidas al efecto de invernadero.

El Protocolo de Kyoto, es el principal acuerdo internacional para el combate a las alteraciones climáticas. Ha entrado en vigencia el 16 de Febrero de 2005 (después de su ratificación por la Federación Rusa), siete años después de su redacción. Portugal ya ha alcanzado en 2002 cerca del $41 \%$ de aumento en sus emisiones comparativamente con el año de referencia, 1990, siendo el límite establecido de 27\% para el período 2008-2012. En consecuencia, Portugal tiene que mejorar la eficiencia de producción de energía eléctrica y utilizar Fuentes de Energía Renovable, FER, para cumplir el límite establecido. En Portugal, según la 'Direcção Geral de Geologia e Energia', DGGE, hasta Noviembre de 2006, la capacidad instalada de potencia para la producción de energía eléctrica utilizando FER, ha alcanzado la cifra de $6905 \mathrm{MW}$, de los cuales $1637 \mathrm{MW}$ son de potencia eólica (DGGE, 2006). A la luz de la Directiva 2001/77/CE, en 2005, las FER representaron el $35 \%$ del consumo bruto de energía eléctrica.

Diversos autores han estudiado modelos matemáticos para los sistemas eólicos, así como la calidad de la energía eléctrica en lo que respecta al contenido armónico. Chen (2001), presenta un trabajo de modelización y simulación para el estudio de la onda de tensión y su distorsión armónica en una red eléctrica donde están conectadas turbinas eólicas, demuestra que la fluctuación en la tensión y el contenido armónico puede ser minimizado utilizando convertidores electrónicos de potencia; y recorriendo a un sistema de control adecuado, permite al sistema eólico extraer la máxima energía cinética del viento.

Slootweg et al. (2001a), estudian modelos para la simulación dinámica de sistemas eólicos, concluye que después de las simplificaciones necesarias en el modelo sub-transitorio, las diferencias entre los generadores síncrono y asíncrono de doble alimentación usados en sistemas eólicos de velocidad variable no son detectadas en su interacción con la red eléctrica debido a la actuación de los controladores. Slootweg et al., (2001b), estudian el generador asíncrono de doble alimentación con convertidor electrónico de potencia conectado al rotor de la máquina, utiliza controladores para el ángulo de paso y velocidad de giro de la turbina y para la tensión a los terminales de la máquina. Datta y Ranganathan (2002), estudian la conexión a la red eléctrica de un sistema eólico de velocidad variable con generador asíncrono de doble alimentación, concluye que con este generador se puede mejorar significativamente la extracción de energía cinética del viento lo que contribuye para bajar el coste de los convertidores electrónicos de potencia, y una mejor utilización del generador comparando con el generador de jaula. Slootweg et al. (2003), estudian el impacto de los diversos sistemas eólicos en la dinámica de la red eléctrica, concluye que simplificando los modelos de los sistemas se reducen los tiempos de simulación.

Achilles y Pöller (2004) estudian un sistema eólico con generador síncrono con convertidores de potencia, comparando los resultados del modelo detallado con el modelo simplificado, concluye que el modelo simplificado produce resultados con una precisión aceptable y reducción de los tiempos de simulación. Blaabjerg et al. (2004) hace una revisión de la aplicación de los convertidores de potencia en los sistemas de generación distribuida, y concluye que el uso de interfaces de electrónica de potencia permite la integración de la generación distribuida de una forma apropiada. Polisetty et al. (2006), presentan el proyecto de un control de coordinación entre un convertidor estático de energía reactiva y un parque eólico, para mejorar el perfil de la onda de tensión y la estabilidad de la red eléctrica, presenta dos metodologías de coordinación basadas respectivamente en el control convencional y en el control neuronal adaptativo, concluye que el control no lineal tiene un comportamiento menos forzado relativamente al lineal.

El aporte del presente trabajo consiste en estudiar y simular en Simulink la aplicación de diferentes topologías de convertidores de potencia en los sistemas de energía eólica: el convertidor de dos niveles, el convertidor multinivel y el convertidor matricial. Los modernos sistemas de 
aprovechamiento de energía eólica de velocidad variable, utilizan como interfaces convertidores electrónicos de potencia, capaces de desacoplar su frecuencia de trabajo con respecto a la frecuencia de la red eléctrica. La utilización de interfaces de electrónica de potencia en los sistemas eólicos permiten mejorar la eficiencia del proceso de conversión de energía, lo que, conjugado con la creciente relación calidad/precio de los equipamientos de electrónica de potencia, permite esperar que su penetración como componente de los sistemas eólicos sea cada vez más significativa. El adecuado control del convertidor de potencia permite establecer la corriente por los devanados y controlar así el par resistente que opone la máquina eléctrica al giro de la turbina. Los semiconductores mayoritariamente empleados en los convertidores de los sistemas eólicos de velocidad variable, son los IGBT's.

Los sistemas con convertidores de dos niveles y multinivel son de dos etapas, es decir, la interface de electrónica de potencia está formada por dos convertidores de potencia de transistores IGBT's para conectar el sistema eólico de velocidad variable a la red eléctrica. El primer convertidor, trabajando como un rectificador, está conectado entre la máquina eléctrica y los condensadores. El segundo convertidor, trabajando como un ondulador, está conectado entre los condensadores y un filtro conectado antes de la red eléctrica. Los sistemas con convertidor matricial son de una sola etapa, es decir, no tienen etapa intermedia de tensión continua, se presentan como una alternativa (Pinto y Silva, 2004) que será estudiada.

En los sistemas con convertidor matricial, la interface de electrónica de potencia está formada por nueve transistores IGBT's (by direccionales). Estas interfaces conectan los sistemas trifásicos de entrada, la máquina eléctrica, y el sistema trifásico de salida, la red eléctrica, obteniéndose así las ondas de corriente y tensión con la frecuencia deseadas.

El sistema eólico es constituido por una turbina eólica de velocidad variable conectada a una máquina eléctrica síncrona, conectada en serie a un filtro de segundo orden y por su vez el filtro está conectado a la entrada del convertidor matricial. Mientras que el sistema trifásico de salida está constituido por la salida del convertidor matricial conectado a la red eléctrica. Los convertidores matriciales han sido objeto de investigación desde finales de los años setenta para ser desarrolladas mejores potencialidades. El inconveniente de estos convertidores está en la distorsión de las ondas de corriente, que se produce cuando las tensiones son desequilibradas, pues no incorporan etapa de tensión continua con condensador, como las topologías de dos niveles y multinivel (Amenedo et al., 2003).

En la simulación se ha optado por la representación de la máquina eléctrica y el sistema de energía eléctrica como una impedancia en serie con una fuente de tensión constante. También en la simulación se ha optado por la representación de los transistores IGBT's y los diodos como interruptores ideales.

\section{CONVERTIDOR DE DOS NIVELES}

En el estudio de los sistemas con convertidores de dos niveles como indicado en la Fig. 1, se considera que: 1) Los transistores IGBT's son ideales, y unidireccionales y jamás estarán sujetos a tensiones inversas, siendo esta situación garantizada por la topología de conexión de los diodos en antiparalelo; 2) Los diodos son ideales: en conducción es nula la caída de tensión a sus terminales y en bloqueo é nula la corriente que pasa; 3 ) La tensión continua en la salida del rectificador debe ser siempre $v_{d c}>0$. En el convertidor de dos niveles, tanto el rectificador como el ondulador están constituidos por seis IGBT's comandados $S_{\mathrm{ik}}$. Cada IGBT esta conectado en antiparalelo a um diodo. Los conjuntos de dos IGBT's conectados a la misma fase constituyen un brazo del convertidor. En el sistema indicado en la Fig. 1, el primer convertidor trabaja como un rectificador. Este está conectado entre la máquina eléctrica cuya representación se hace por un circuito con la inductancia $L$, la resistencia $R$ y una fuente de tensión $u_{k}$ para $\mathrm{k}=\{1,2,3\}$ y el banco de condensadores $\mathrm{C}$. El segundo convertidor trabaja como un ondulador, está conectado entre el banco de condensadores y un filtro inductivo cuya inductancia es $L_{f}$ conectado antes de la red eléctrica cuya representación se hace por un circuito cuya inductancia es $L_{c}$, la resistencia es $R_{c}$ y una fuente de tensión $u_{k}$ para $k=\{4,5,6\}$. 


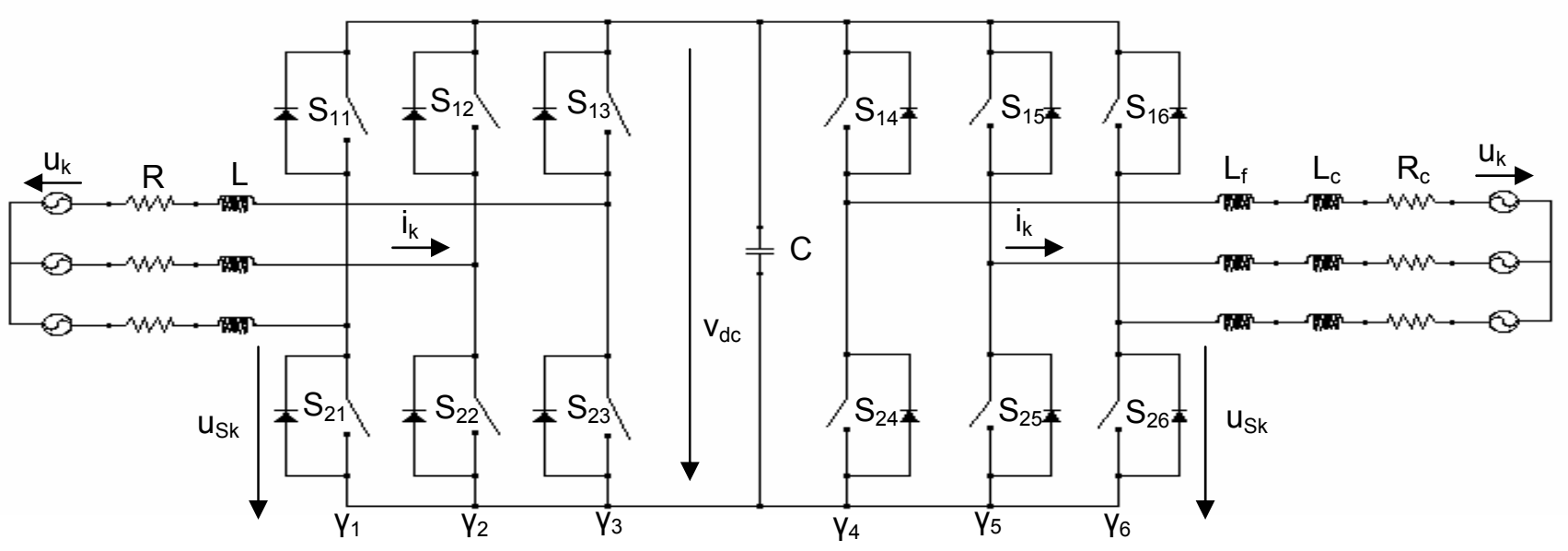

Fig. 1: Sistema con convertidor de dos niveles

Para la función de conmutación de cada IGBT, se utiliza una variable binaria $\gamma_{k}$ para identificar el estado del IGBT en el brazo k del convertidor, siendo k el índice que identifica el brazo. Siendo $S_{i k}$ los estados lógicos de conducción o bloqueo de los IGBT's i de cada brazo k del convertidor, los dos IGBT's son identificados por el índice i con los valores 1, para el IGBT superior y 2 para el IGBT inferior según la Fig. 1. Así, para simular los estados de conducción y bloqueo de los IGBT's, de cada brazo k de los convertidores, con $k=\{1,2,3\}$ para el rectificador y $k=\{4,5,6\}$ para el ondulador, se establecen las condiciones lógicas siguientes:

$\mathrm{V}_{\mathrm{k}}= \begin{cases}1, & \mathrm{~S}_{\mathrm{ik}}=1 \text { (conducción) } \\ 0, & \mathrm{~S}_{\mathrm{ik}}=0 \text { (bloqueo) }\end{cases}$

para $\mathrm{i} \in\{1,2\}$ y $\mathrm{k} \in\{1, \ldots, 6\}$

Además se establece la siguiente restricción topológica para los brazos;

$\sum_{\mathrm{i}=1}^{2} \mathrm{~S}_{\mathrm{ik}}=1$ para $\mathrm{k} \in\{1, \ldots, 6\}$

La tensión simple $\mathrm{u}_{\mathrm{sk}}$ a los terminales del rectificador y del ondulador dependen directamente de los estados de los transistores IGBT's. Las corrientes en el rectificador son determinadas por las ecuaciones de estado,

$\frac{\mathrm{di}_{\mathrm{k}}}{\mathrm{dt}}=-\frac{\mathrm{R}}{\mathrm{L}} \mathrm{i}_{\mathrm{k}}+\frac{1}{\mathrm{~L}} \mathrm{u}_{\mathrm{k}}-\frac{1}{\mathrm{~L}} \mathrm{u}_{\mathrm{sk}}$

para $k=\{1,2,3\}$

Las corrientes en el ondulador son determinadas por las ecuaciones de estado,

$\frac{d_{k}}{d t}=\frac{1}{\left(L_{c}+L_{f}\right)}\left(u_{s k}-u_{k}-R_{c} i_{k}\right)$

para $\mathrm{k}=\{4,5,6\}$

y la tensión continua es determinada por la ecuación de estado

Así, (1) a (5) describen el modelo de estado del convertidor de dos niveles.

$\frac{d v_{d c}}{d t}=\frac{1}{C}\left(\sum_{k=1}^{3} v_{k} i_{k}-\sum_{k=4}^{6} v_{k} i_{k}\right)$ 


\section{CONVERTIDOR MULTINIVEL}

En el sistema con convertidor multinivel, el rectificador está constituido por doce transistores IGBT's comandados $S_{i k}$. En el ondulador la constitución es la misma. En este sistema, el primer convertidor trabaja como un rectificador. Está conectado entre la máquina eléctrica cuya representación se hace por un circuito con la inductancia $L$, la resistencia $R$ y una fuente de tensión $u_{k}$ para $k=\{1,2,3\}$ y los bancos de condensadores $\mathrm{C}_{1}$ y $\mathrm{C}_{2}$. El segundo convertidor trabaja como un ondulador, está conectado entre los bancos de condensadores y un filtro de segundo orden cuya inductancia es $L_{f}$, con la resistencia $R_{f}$ y un banco de condensadores $C_{f}$ conectado antes de la red eléctrica. La red eléctrica se representa por un circuito cuya inductancia es $L_{c}$, la resistencia $R_{c}$ y una fuente de tensión $u_{k}$ para $k=\{4,5,6\}$. La configuración del sistema que será simulada se presenta en la Fig. 2. Los conjuntos de cuatro transistores IGBT's conectados a la misma fase constituyen el brazo k del convertidor.

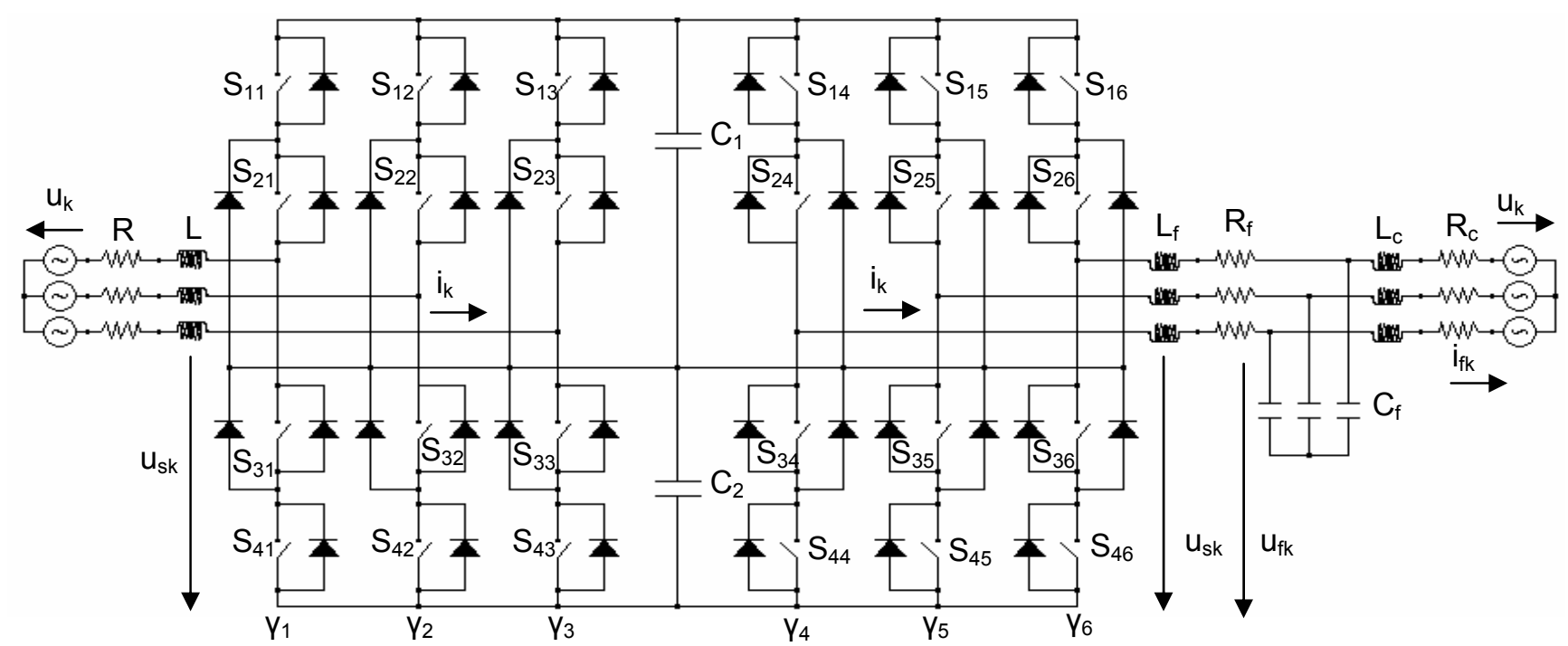

Fig. 2: Sistema con convertidor multinivel

En el estudio del convertidor multinivel se hacen las mismas consideraciones 1), 2) y 3) hechas para los convertidores de dos niveles. $\mathrm{Y}$ además, se hace la siguiente consideración: cada brazo $\mathrm{k}$ del convertidor debe tener siempre funcionando dos IGBT's. Para la función de conmutación de cada IGBT $S_{i k}$ se utiliza la variable binaria $\gamma_{k}$ para identificar el estado de conducción y bloqueo del IGBT i en el brazo $\mathrm{k}$ del convertidor, siendo $\mathrm{i}$ el índice que identifica el IGBT con $\mathrm{i}=\{1,2,3,4\}$ el $\mathrm{k}$ el índice que identifica el brazo. Así, para simular los estados de conducción y bloqueo de los IGBT's, de cada brazo k de los convertidores, con $k=\{1,2,3\}$ para el rectificador y $k=\{4,5,6\}$ para el ondulador, se establecen las condiciones lógicas siguientes:

$V_{k}=\left\{\begin{array}{cc}1, & \left(S_{1 k} \text { y } S_{2 k}\right)=1 \\ 0, & \left(S_{2 k} \text { y } S_{3 k}\right)=1 \\ -1, & \left(S_{3 k} \text { y } S_{4 k}\right)=1\end{array}\right.$

para $\mathrm{k} \in\{1, \ldots, 6\}$

Y además, se establece la siguiente restricción topológica para los brazos,

$\left(S_{1 k} \cdot S_{2 k}\right)+\left(S_{2 k} \cdot S_{3 k}\right)+\left(S_{3 k} \cdot S_{4 k}\right)=1$

para $\mathrm{k} \in\{1, \ldots, 6\}$

Se obtiene en cada brazo k para los dos IGBT's superiores $\left(S_{1 k}\right.$ y $\left.S_{2 k}\right)$ una variable binaria equivalente $\Gamma_{1 \mathrm{k}}$ y para los dos IGBT's inferiores $\left(S_{3 k}\right.$ y $\left.S_{4 k}\right)$ la variable binaria equivalente $\Gamma_{2 k}$. La matriz de comando del rectificador depende directamente de los estados de conducción y bloqueo de los IGBT's. 
$\Gamma_{1 \mathrm{k}}=\frac{\mathrm{Y}_{\mathrm{k}}\left(1+\mathrm{Y}_{\mathrm{k}}\right)}{2} ; \Gamma_{2 \mathrm{k}}=\frac{\mathrm{Y}_{\mathrm{k}}\left(1-\mathrm{Y}_{\mathrm{k}}\right)}{2}$

para $\mathrm{k} \in\{1, \ldots, 6\}$

Las corrientes en el rectificador son determinadas por la ecuación,

$\frac{\mathrm{di}_{\mathrm{k}}}{\mathrm{dt}}=\frac{1}{\mathrm{~L}}\left(\mathrm{u}_{\mathrm{k}}-\mathrm{R} \mathrm{i}_{\mathrm{k}}-\mathrm{u}_{\mathrm{sk}}\right)$

para $\mathrm{k}=\{1,2,3\}$

Las corrientes en el filtro de segundo orden son determinadas por la ecuación de estado,

$\frac{\mathrm{di}_{\mathrm{fk}}}{\mathrm{dt}}=\frac{1}{\mathrm{~L}_{\mathrm{c}}}\left(\mathrm{u}_{\mathrm{k}}-\mathrm{R}_{\mathrm{c}} \mathrm{i}_{\mathrm{fk}}-\mathrm{u}_{\mathrm{fk}}\right)$

para $k=\{4,5,6\}$

La tensión continua es la suma de las tensiones en los condensadores $C_{1}$ y $C_{2}$ es determinada por la ecuación de estado,

$$
\begin{aligned}
& \frac{d v_{d c}}{d t}=\frac{1}{C_{1}}\left(\sum_{k=1}^{3} \Gamma_{1 k} i_{k}-\sum_{k=4}^{6} \Gamma_{1 k} i_{k}\right)+ \\
& +\frac{1}{C_{2}}\left(\sum_{k=1}^{3} \Gamma_{2 k} i_{k}-\sum_{k=4}^{6} \Gamma_{2 k} i_{k}\right)
\end{aligned}
$$

Las ecuaciones (6) a (11) describen el modelo de estado del convertidor multinivel.

\section{CONVERTIDOR MATRICIAL}

El modelo de estado del sistema con convertidor matricial de acuerdo con (Pinto y Silva, 2004), es definido como una asociación de nueve interruptores by direccionales $S_{i k}$ que interconectan dos sistemas trifásicos. La configuración del sistema que será simulada se presenta en la Fig. 3

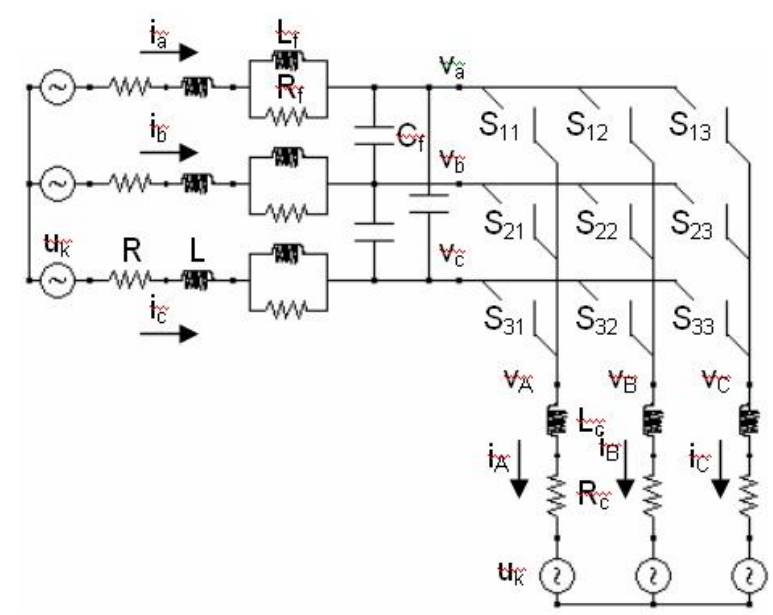

Fig. 3: Sistema con convertidor matricial

El sistema trifásico de entrada, constituido por una máquina eléctrica cuya representación se hace por un circuito con la inductancia $L$, la resistencia $R$ y una fuente de tensión $u_{k}$ para $k=\{a, b, c\}$, conectada $a$ un filtro de segundo orden cuya inductancia es $L_{f}$, con la resistencia $R_{f}$ y un banco de condensadores $C_{f}$ y el sistema trifásico de salida se representa por un circuito cuya inductancia es $L_{c}$, la resistencia $R_{c}$ y una fuente de tensión $u_{k}$ para $k=\{A, B, C\}$. En el estudio del convertidor matricial se considera que: 1) Los diodos son ideales: en conducción es nula la caída de tensión a sus terminales y en bloqueo es nula la corriente que pasa; 2) Cada elemento de la matriz de comando [S] del convertidor es un interruptor by 
direccional en tensión y corriente; 3) Para que haya siempre continuidad en la corriente, en cada línea de la matriz de comando [S] existirá una variable de comando que deberá corresponder a un interruptor en conducción; 4) Para que no haya la posibilidad de cortocircuito con las fuentes de tensión, en cada línea de la matriz de comando [S] no habrá mas que una variable de comando correspondiente a un interruptor en conducción. Así, en el convertidor matricial, se establecen las condiciones lógicas para los IGBT's,

$\mathrm{S}_{\mathrm{ij}}=\left\{\begin{array}{l}1, \text { (conducción) } \\ 0, \text { (bloqueo })\end{array}\right.$ para $\mathrm{i}, \mathrm{j} \in\{1,2,3\}$

Las consideraciones 3) y 4) se expresan por la expresión lógica,

$\sum_{\mathrm{j}=1}^{3} \mathrm{~S}_{\mathrm{ij}}=1 \quad$ para $\mathrm{i} \in\{1,2,3\}$

Las tensiones simples de salida están conectadas con las tensiones simples de entrada del convertidor por la matriz de comando [S] dadas por,

$\left[\begin{array}{l}v_{A} \\ v_{B} \\ v_{C}\end{array}\right]=\left[\begin{array}{lll}S_{11} & S_{12} & S_{13} \\ S_{21} & S_{22} & S_{23} \\ S_{31} & S_{32} & S_{33}\end{array}\right]\left[\begin{array}{l}v_{a} \\ v_{b} \\ v_{c}\end{array}\right]=[S]\left[\begin{array}{l}v_{a} \\ v_{b} \\ v_{c}\end{array}\right]$

La relación entre las corrientes de entrada en el convertidor y las corrientes en la red de energía eléctrica vienen dadas por,

$\left[\begin{array}{lll}i_{a} & i_{b} & i_{c}\end{array}\right]^{\top}=[S]^{\top}\left[\begin{array}{lll}i_{A} & i_{B} & i_{C}\end{array}\right]^{\top}$

Las ecuaciones (12) a (15) describen el convertidor matricial.

\section{ASPECTOS DEL CONTROL}

Los controladores utilizados en los convertidores son del tipo PI. Para el comando de los IGBT's de las tres topologías de convertidores, se ha utilizado el método de la modulación de los pulsos de disparo mediante vectores espaciales de tensión SVM (space vector modulation) y el control por modo de deslizamiento. Se obligan a las grandezas que se pretenden controlar a seguir referencias impuestas al sistema que se quiere comandar, permitiendo escoger las combinaciones de conexiones de interruptores más adecuadas, obteniéndose así las ondas de corriente y tensión con la frecuencia deseadas.

La representación de los vectores espaciales de la tensión se hace en el plano $\alpha \beta$ utilizando la transformación de Concórdia (Silva, 2001). Los semiconductores de potencia presentan limitaciones físicas que no les permite frecuencias de conmutación elevadas. Así, para un determinado valor de frecuencia de conmutación, va existir un error $e_{\alpha \beta}$ entre las grandezas de referencia y las grandezas que se pretenden controlar en el plano $\alpha \beta$ por lo que no se puede anular la condición de estabilidad del modo de deslizamiento (Silva, 2001).

$S\left(e_{\alpha \beta}, t\right) \frac{d S\left(e_{\alpha \beta}, t\right)}{d t}<0$

Para hacer control en los convertidores se define un error $\pm \varepsilon$ con un valor suficientemente pequeño, teniendo entonces que se satisfacer bandas de error.

$-\varepsilon<S\left(e_{\alpha \beta}, t\right)<+\varepsilon$ 
Mediante la utilización de comparadores de histerese en Simulink, se tiene la garantia que las funciones de conmutación se encuentran dentro de bandas de error pretendidas. En el caso del convertidor matricial, las tensiones compuestas de salida del convertidor no son variables de estado. Como la frecuencia de conmutación de los interruptores es muy superior a la frecuencia de las tensiones a controlar, se considera que durante un periodo de conmutación $T_{S}$ el valor medio de las tensiones compuestas a controlar $V_{\alpha \beta}$ es igual al valor medio de las tensiones compuestas de referencia de la red eléctrica $V_{\alpha \beta}^{*}$

$\frac{1}{T_{s}} \int_{n T_{s}}^{(n+1) T_{s}}\left(v_{\alpha \beta}^{*}-v_{\alpha \beta}\right) d t=0$

De esta forma, es posible determinar las funciones de conmutación que establecen la superficie de deslizamiento $S\left(e_{\alpha \beta}, t\right)$ necesaria para controlar las tensiones compuestas de salida del convertidor matricial.

\section{RESULTADOS}

Se han implementado los modelos matemáticos de los convertidores en Simulink. Así, en la simulación del modelo del convertidor de dos niveles descrito por las ecuaciones (1) al (5), se obtuvieron las ondas de tensión en la salida del ondulador $u_{\text {sk }}$ para $k=\{4,5,6\}$ que se presentan en la Fig. 4.

Las ondas de corriente en la salida del ondulador $\mathrm{i}_{\mathrm{k}}$ para $\mathrm{k}=\{4,5,6\}$ se presentan en la Fig. 5.

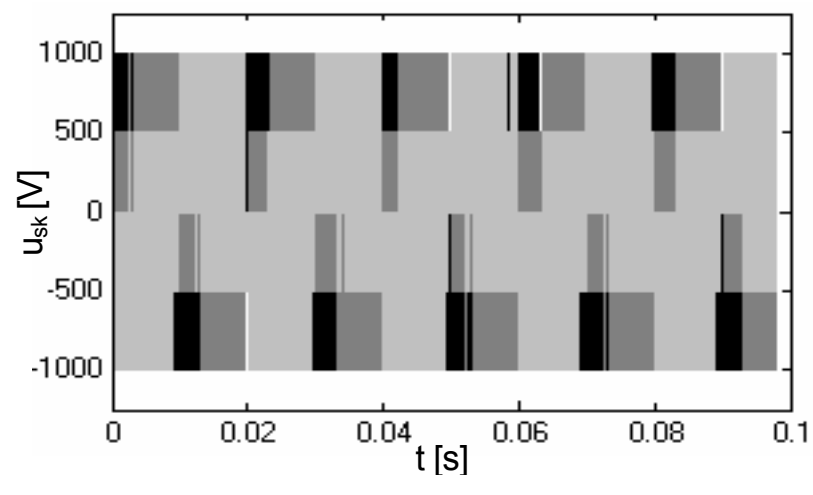

Fig. 4: Ondas de tensión $u_{\text {sk }}$ para $k=\{4,5,6\}$

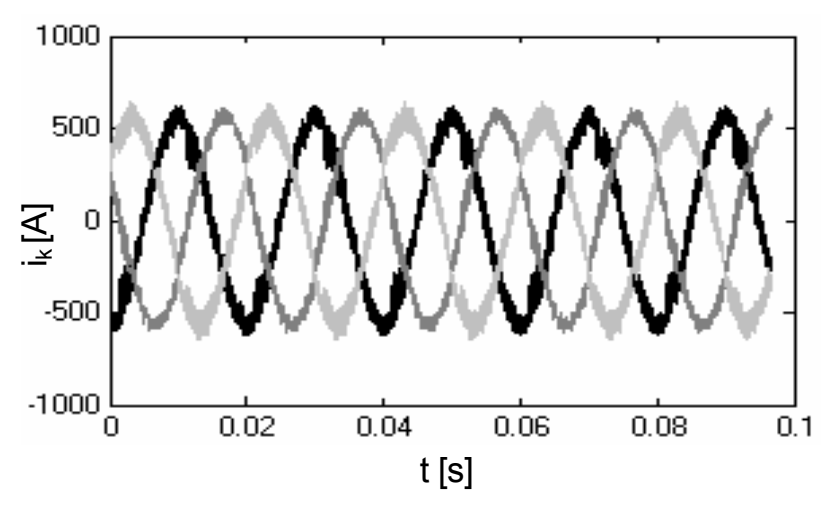

Fig. 5: Ondas de corriente $\mathrm{i}_{\mathrm{k}}$ para $\mathrm{k}=\{4,5,6\}$

En la simulación del modelo del convertidor multinivel descrito por las ecuaciones (6) al (11), se obtuvieron las ondas de tensión en el filtro de segundo orden $u_{\mathrm{fk}}$ para $k=\{4,5,6\}$ que se presentan en la Fig. 6.

En la simulación del modelo del convertidor matricial descrito por las ecuaciones (12) al (15), se obtuvieron las ondas de tensión en la salida del ondulador $\mathrm{v}_{\mathrm{ABC}}$ se presentan en la Fig. 8.

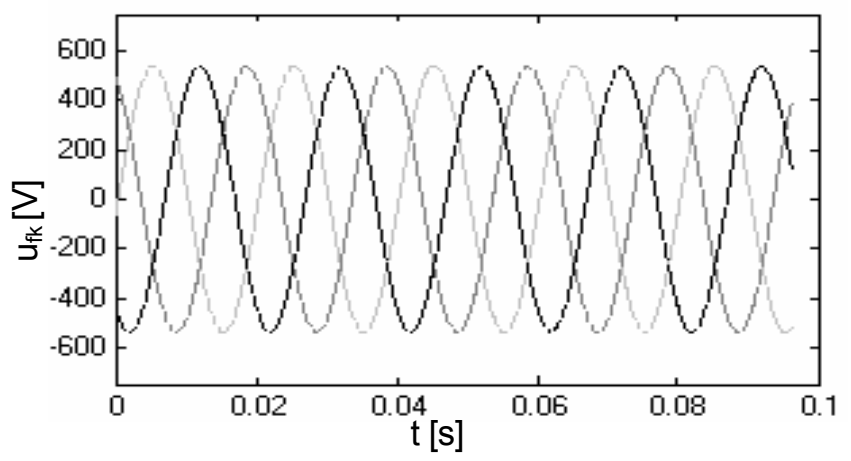

Fig. 6: Ondas de tensión $u_{\mathrm{fk}}$ para $\mathrm{k}=\{4,5,6\}$

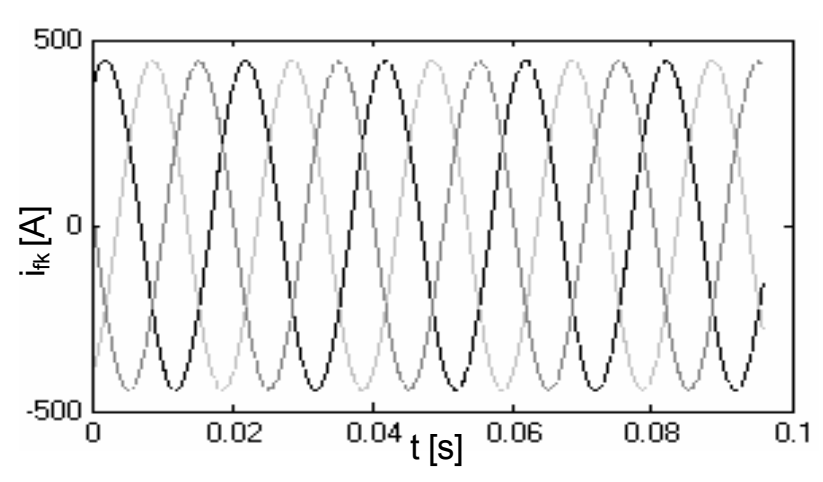

Fig. 7: Ondas de corriente $\mathrm{i}_{\mathrm{fk}}$ para $\mathrm{k}=\{4,5,6\}$

Las ondas de corriente en la salida del ondulador $\mathrm{i}_{\mathrm{fk}}$ para $\mathrm{k}=\{4,5,6\}$ se presentan en la Fig. 7. 


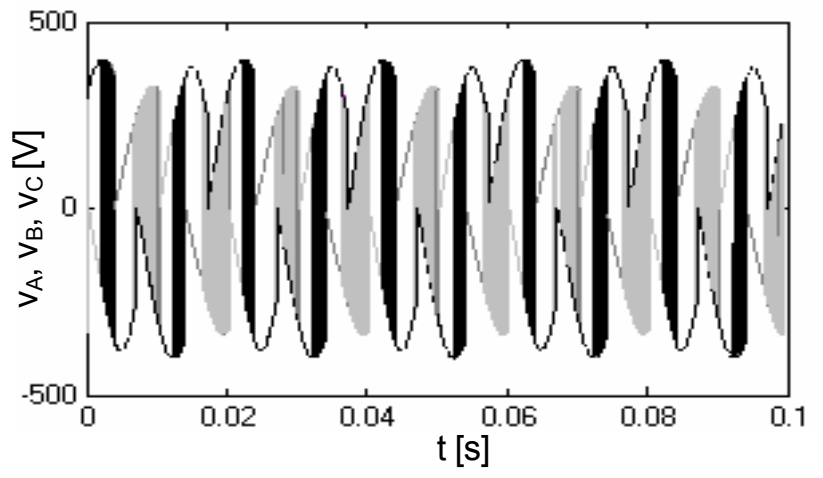

Fig. 8: Ondas de tensión $v_{A}, v_{B} y v_{C}$

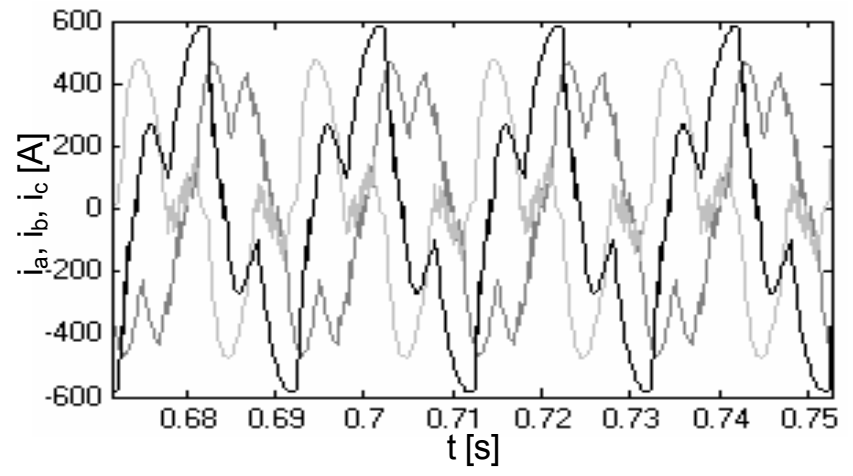

Fig. 9: Ondas de corriente $i_{a}, i_{b} y i_{c}$

Las ondas de corriente en la entrada del convertidor $\mathrm{i}_{\mathrm{a}}, \mathrm{i}_{\mathrm{b}}$ y $\mathrm{i}_{\mathrm{c}}$ se presentan en la Fig. 9.

No es necesario hacer análisis armónica en las ondas de tensión y corriente, para concluir que las ondas de tensión y corriente son diferentes en los tres convertidores, una vez que, los resultados obtenidos en las Fig. 4 a Fig. 9 permiten por observación visual esta conclusión. De cualquier forma las ondas de tensión y corriente en los convertidores de dos etapas se presentan con mejor comportamiento armónico que en el convertidor de una etapa, es decir el convertidor matricial.

\section{CONCLUSIONES}

De los resultados presentados, se pueden obtener las siguientes conclusiones: 1) El convertidor multinivel con filtro de segundo orden es el que presenta ondas de tensión y corriente en el punto de inyección en la red de energía eléctrica con mejor comportamiento armónico; 2) el convertidor matricial siendo de una sola etapa presenta ondas de tensión y corriente con peor comportamiento armónico; y 3) el comportamiento armónico de la onda de tensión (inyectada en la red eléctrica) en el convertidor de dos niveles, Fig. 4 se debe a la frecuencia de conmutación de los IGBT's.

\section{REFERENCIAS}

Achilles, S. y M. Pöller; Direct Drive Synchronous Machine Models for Stability Assessment of Wind Farms, DIgSILENT GmbH, Heinrich-Hertz-Str. 9, 72810 Gomaringen, Germany (2004).

Amenedo, J.L., J.C. Díaz y S. Gómez; Sistemas Eólicos de Producción de Energía Eléctrica. Rueda, Madrid, España (2003).

Blaabjerg, F., Z. Chen y S.B. Kjaer; Power Electronics as Efficient Interface in Dispersed Power Generation Systems, IEEE Transactions on Energy Convertion: 19(5) (2004).

Chen, Z.; Grid Power Quality with Variable Speed Wind Turbines, IEEE Transactions on Energy Convertion: 16(2) (2001).

Datta, R. y V.T. Ranganathan; Variable Speed Wind Power Generation Using Doubly Fed Wound Rotor Induction Machine - A Comparison with Alternative Schemes, IEEE Transactions on Energy Convertion: 17(3) (2002).

DGGE; Energias Renováveis Estatísticas Rápidas - n²1, Lisboa, Portugal (2006).

Pinto S., J.F. Silva; "Direct Control Method for Matrix Converters with Input Power Factor Regulation", IEEE Proc. PESC 2004 (CD ROM), 2366-2372, Aachen, Alemania, 20 al 26 Junio (2004).

Polisetty, V.K. y otros tres autores; Intelligent Integration of a Wind Farm to an Utility Power Network with Improved Voltage Stability, IEEE Industry Applications Conference, 3, 1128-1133, Tampa, USA Octubre (2006). 
Silva, F.; Control Methods for Power Converters, Power Electronics Handbok, M. Rashid Academic Press, USA (2001).

Slootweg, J.G. y otros tres autores; Modelling Wind Turbines in Power System Dynamics Simulations, IEEE Power Ingineering Society, 1, 22-26, 15 al 19 Julio (2001a).

Slootweg, J.G., H. Polinder y W.L. Kling; Dynamic Modelling of a Wind Turbine with Doubly Fed Induction Generator, IEEE Power Ingineering Society, 1, 644-649, 15 al 19 Julio (2001b).

Slootweg, J.G., H. Polinder y W.L. Kling; Representing Wind Turbine Electrical Generating Systems in Fundamental Frequency Simulations, IEEE Transactions on Energy Convertion: 18(4) (2003). 